Erschienen in: Deppermann, Arnulf/Linke, Angelika (Hrsg.): Sprache

intermedial. Stimme und Schrift, Bild und Ton. - Berlin/New York: de

Gruyter, 2010. S. 271-298. (Jahrbuch des Instituts für Deutsche Sprache 2009), https://doi.org/10.1515/9783110223613.271

Peter Auer

\title{
Sprachliche Landschaften
}

\section{Die Strukturierung des öffentlichen Raums durch die geschriebene Sprache ${ }^{1}$}

\begin{abstract}
Es gehört zu den Gemeinplätzen der Linguistik, dass gesprochene Sprache situationsgebunden, geschriebene Sprache aber zumindestens tendenziell situationsgelöst sei und sich über Orte und Zeitpunkte hinweg transportieren lässt, ohne ihre Bedeutung wesentlich zu verändern. Eine große Menge von schriftlichen Zeichen ist allerdings genau durch das umgekehrte Phänomen gekennzeichnet: sie sind ortsstabil und in ihrer Bedeutung von ihrem lokalen Kontext abhängig. Die Schrift auf Schildern, Plakaten und anderen öffentlichen Zeichen ist eine spezifische Form von Sprache, die den gängigen Stereotype von Schriftlichkeit widerspricht, weil sie „ding"“- bzw. „ortsfest" ist und funktional wie auch formal anderen Regelmäßigkeiten folgt als die meist betrachtete Schrift der Texte auf transportablen Trägern wie Buch, Zeitung, elektronischem Textdokument oder e-mail. Solche Funktionen von Schrift (wie Wegweisen, Orientierung geben, Erinnern und Appellieren, Zugehörigkeit signalisieren) sind seit deren Erfindung relevant gewesen; erst in jüngster Zeit werden aber orts- und dingfesten Zeichen unter dem Begriff der linguistic landscapes ins Blickfeld der Linguistik gerückt und systematisch untersucht. Das Forschungsinteresse liegt vor allem in der Beantwortung der Frage, wie öffentliche Zeichen Sprach-Räume konstituieren, und zwar insbesondere monolinguale oder bilinguale Räume in mehrsprachigen Gesellschaften. Der folgende Beitrag geht aber nur nebenbei auf solche Sprach-Räume ein; sein primäres Interesse gilt der Art und Weise, wie öffentliche, orts- und dingfeste Zeichen überhaupt Raum konstituieren, d.h. wie wir semiotisch dicht organisierte Räume ,lesen', um uns in ihnen zu orientieren.
\end{abstract}

\section{Die Mär von der Situationsunabhängigkeit der geschriebenen Sprache}

Es gehört zu den Allgemeinplätzen der Sprachwissenschaft, dass sich gesprochene und geschriebene Sprache durch ihren Bezug auf die Situation unterscheiden: während die gesprochene Sprache, wie vielleicht am prominentesten Karl Bühler dargelegt hat (Bühler 1934 [1982]), von der Sprechsituation - also der zeitlichen (HIC), räumlichen (NUNC) und personalen (EGO) Verankerung des Sprechakts - abhängt, sei die geschriebene Sprache von Zeit, Ort und Sprecher losgelöst und auf diese Weise transportabel, also situationsunabhängig verständlich. Das ist ohne Zweifel für einen großen Teil der schriftsprachlichen Produkte in Vergangenheit und Gegen-

Mein Dank für Kommentare und Hinweise geht an Karin Birkner, Arnulf Deppermann sowie Anja Stukenbrock. Einige der verwendeten Fotos gehen auf fieldwork von Studierenden des Studiengangs Master of European Linguistics (MEL) an der Universität Freiburg zurück. Auch ihnen sei hiermit gedankt! 
wart zutreffend, und es lässt sich daraus zu Recht ableiten, dass Schriftsprache ein Verfahren ist, das gesellschaftliche Gedächtnis zu objektivieren. Schrifttexte in Medien wie Büchern, Zeitungen oder dem Internet sind dazu geeignet, immer wieder (von unterschiedlichen Menschen an unterschiedlichen Orten und zu unterschiedlichen Zeitpunkten) gelesen zu werden. Diese situationsunabhängige Stabilität prädestiniert sie, den Wissenskanon einer Gesellschaft zu formen. Zu diesem Kanon gehören in ganz besonderer Weise auch normative Texte. Schrift etabliert so Traditionen und dadurch auch abstrakte gesellschaftliche Institutionen wie das Recht; sie sorgt für gesellschaftliche Stabilität (Ong 1982). Dazu trägt neben der Transportabilität der Texte auch ihre Reproduzierbarkeit bei, die von handgeschriebenen Textrollen und Büchern über Inkunablen und gedruckte Bücher bis zu elektronischen Texte in mehreren technologischen Revolutionen immer wieder radikal verbessert wurde.

Das ist jedoch nur ein Teil der Wahrheit, und nur ein Teil der Geschichte und Funktionalität der Schrift. Daneben gibt es eine andere Funktionalität, die mindestens ebenso alt ist, in der Forschung aber oft vergessen wird. In dieser Verwendung ist die Schrift unauflöslich mit dem Objekt, auf dem sie angebracht ist, oder dem Ort, an dem sich dieses Objekt befindet, verbunden, und kann ohne diese Dingfestigkeit und/oder Lokalisierung gar nicht verstanden werden. Schrift ist hier zwar vom Sprecher und von der Zeit gelöst, sie ist jedoch räumlich bzw. dinglich verankert. Bühler (1934 [1982], S. 159) erfasst einen Teil dieser Schriftfunktion, wenn er von symphysischen Zeichen spricht, die durch ihre „dingliche Anheftung“ gekennzeichnet sind. Dass es sich hier ebenfalls um eine der originären und primären Funktionen von Schrift handelt, bezeugen viele Schriftdokumente aus beginnenden und frühen Schriftkulturen. Eines der ältesten Schriftdokumente des Germanischen aus dem ansonsten kaum dokumentierten Nordwestgermanisch sind zum Beispiel die Hörner von Gallehus, die vermutlich anlässlich einer Sonnenfinsternis des Jahres 413 angefertigt wurden. Auf einem Horn befindet sich in Runeninschrift der Satz

\section{EK HLEWAGASTIZ HOLTIJAZ HORNA TAWIDO}

„Ich, Hlewagast, der Holte, habe das Horn gemacht“.

Es ist offensichtlich, dass die Schrift hier der Zuordnung eines Objekts zu einer Person (vermutlich des Auftraggebers) dient - eine der grundlegenden Funktionen von dingfester Schriftsprache. Der Satz löst zwar das deiktische Pronomen $e k$ durch Namensnennung auf. Die Nominalphrase borna lässt sich aber nicht referenziell interpretieren, wenn nicht das Objekt bekannt ist, auf das sie sich bezieht: nämlich auf das Horn, auf dem die Schrift angebracht ist. ${ }^{2}$

2 Eine moderne Form solcher dingfester Schriftverwendung untersucht Hausendorf (im Druck) - nämlich das „Kleingedruckte“ auf der Unterseite von Plastiktüten (Warning! Keep 
Auch ortsfeste Inschriften sind in den Schriftkulturen des Altertums weit verbreitet. Auf den ägyptischen Tempeln sind sie in der Regel mit Bildern verbunden. So steht in der Nordwestecke des Vestibüls der HathorKapelle im Hatschepsut-Tempel in Luxor aus dem 15. Jahrhundert v. Chr. die Königin Hatschepsut vor Hathor; zwischen beiden ist noch der Rest der erläuternden Inschrift zu sehen, die wohl vollständig lautete (erhalten ist nur der kursiv gedruckte Teil): „Der König von Ober- und Unterägypten [nämlich Tutmosis] hat ihr(?) Denkmal gemacht für ihre(?) Mutter Hathor, die Herrin von Dendera, sie (!) hat es gemacht [damit ihr] Leben gegeben werde wie Ra."

Die Inschrift ist dingfest (in Stein eingemeißelt), aber auch ortsfest: ihr Bezug auf den Tempel, auf dem sie steht, ist unerlässlich für die richtige Interpretation. Die Funktion der (In-)Schrift ist eine doppelte: einerseits errichtet Tutmosis ein Denkmal für seine Frau Hatschepsut (Ehrungsfunktion), andererseits wird der Tempel der Göttin Hathor geweiht (Weihefunktion). Die Ermahn- und Gedenkfunktion (unter die man die Ehrungsfunktion einordnen kann) ist für die öffentliche, orts- und dingfeste Schriftsprache bis heute typisch, die Weihe- oder Zueignungsfunktion ist hingegen heute selten.

Die beiden Beispiele belegen wie viele andere, dass die These, die Erfindung der Schrift sei essentiell mit der Situationsablösung von Sprache verbunden, nicht zutreffen kann - zumindest nicht in Bezug auf die Dimension des Orts. Die Zurschaustellung von Schrift im öffentlichen Raum ist vielmehr, wie Coulmas jüngst (2008) unter anderem am Beispiel des Codex Hammurabi und des Rosetta Stone zeigt, von grundsätzlicher Bedeutung, wenn man schriftlich fixierte Sprache verstehen möchte. Sie brachte eine wichtige Veränderung der Semiotik des menschlichen Lebensraums mit sich, die eng mit der Entwicklung urbaner Gesellschaften zusammen hängt.

Erst seit etwa 10 Jahren gibt es in der Linguistik eine Forschungsrichtung, die sich mit dieser ding- und vor allem ortsfesten Schriftverwendung im öffentlichen Raum beschäftigt und unter dem etwas irreführenden Begriff linguistic landscape research bekannt geworden ist (vgl. jüngst: Shohamy/ Gorter (Hg.) 2008). Sprachliche Landschaften werden in dieser Forschungstradition meist unter Rückgriff auf einen der ersten Beiträge zu diesem Thema eher aufzählend als definitorisch beschrieben:

The language of public road sings, advertising billboards, street names, place names, commercial shop signs, and public signs on government buildings combines to form the linguistic landscape of a given territory, region, or urban agglomerate. (Landry/Bourhis 1997, S. 25)

Es geht in den bisher publizierten Arbeiten praktisch immer um die Ökologie städtischer Räume, die durch die in ihnen öffentlich sichtbaren Zeichen

this bag away from babies and children!, u.Ä.). 
konstituiert oder zumindest gedeutet werden (vgl. aber Auer 2009). Dabei wurden in der überwiegenden Anzahl der Untersuchungen vor allem multilinguale Gesellschaften untersucht. Diese Einschränkung ist aber keineswegs zwingend; es bleibt sogar offen, bis zu welchem Grad und in welcher Art uns Untersuchungen der sprachlichen Landschaft tatsächlich helfen können, die Struktur einer mehrsprachigen Gemeinschaft besser zu verstehen. Zugleich fällt ein Mangel an theoretischer Reflexion auf, der nur selten zum Beispiel in Ron und Suzie Wong Scollons Arbeit über Discourse in Public Places (2003) - überwunden wird. Ich will im Folgenden eine über den Anwendungsaspekt der multilingualen Städte hinausgehende Orientierung in diesem neuen Forschungsgebiet geben und anhand von Beispielen aus ganz unterschiedlichen soziolinguistischen Kontexten grundsätzliche Klärungen versuchen. Berücksichtigt wird nur die Schnittmenge aus ortsgebundener Schrift (wie oben skizziert) und öffentlichem Sprachgebrauch. Es kommen im öffentlichen Sprachgebrauch durchaus auch andere Schriftzeichentypen vor als die symphysischen; umgekehrt sind nicht alle dingfesten Schriftverwendungen auch öffentlich.

\section{Der öffentliche Gebrauch der Sprache und die Ortsfestigkeit der Schrift}

\subsection{Wozu ortsgebundene Schrift?}

Wie stark öffentliche Räume durch Schrift bestimmt sein können, merken wir vor allem, wenn wir uns in einem Raum bewegen, der zwar offensichtlich durch Schriftzeichen geprägt ist (oder zumindest von ihnen gedeutet wird), in dem wir uns aber schrift-los zurecht finden müssen (etwa in einer japanischen Innenstadt). Andererseits ist offensichtlich, dass es Grade der sprachlichen Konstitution von Räumen gibt. Es scheint etwa nicht weiter erklärungsbedürftig, dass in ländlichen Regionen weniger Schriftzeichen zu sehen sind als in städtischen. Selbst in alten, stark vom Menschen geformten, aber dennoch ruralen Kulturlandschaften wird der Raum lediglich durch Wege, Landmarken und manchmal auch nicht-sprachliche Zeichen (,Steinmännchen'; vgl. Schmaucks 2002) strukturiert. Das deutet bereits auf eine erste wesentliche Eigenschaft der Kommunikation durch öffentliche, ortsgebundene Schrift hin: sie macht vor allem solche Räume les- und damit nutzbar, die nicht durch das routinemäßige Zusammenleben Ortskundiger gekennzeichnet sind. Diese Behauptung impliziert eine spezifische Sichtweise auf den Raum als Möglichkeit sozialen Handelns, wie sie etwa von Anthony Giddens (1984, Kap. 3 und [1979] 1995) entwickelt wird. Danach bedienen sich die Gesellschaftsmitglieder des Raums, um routinemäBig ablaufende Handlungen zu ermöglichen: der Raum bietet Schauplätze 
(locales), die bereits die Möglichkeit bestimmter Aktions- bzw. Interaktionsroutinen in sich tragen. Solange jeder weiß, welche Handlungen an welchen Orten möglich, unmöglich, erwünscht, notwendig, anzuraten etc. sind, ist es nicht notwendig, Schrift einzusetzen, um diese Handlungsräume zu eröffnen. Es ist ja allen bekannt, dass der Weg zu den Feldern an jenem Berg vorbei und an diesem Baum links abbiegend erreicht wird; dass es gefährlich ist, diesem glitschigen Abhang zu nahe zu kommen; dass hier Schutz vor einem Gewitter möglich ist; dass an jener Biegung ein Platz ist, um die Schafe weiden zu lassen etc. Die öffentliche Verwendung der Schrift wird dann relevant, wenn der Raum sich nicht ohne Weiteres in solche allgemein bekannte Orte routinehaften Handelns gliedert, sondern für bestimmte Nutzer erst interpretiert und Handlungstypen zugewiesen werden muss: sei es, dass die Handlungen zwar selbst routinisiert sind, die Nutzer aber die ihnen entsprechenden Orte nicht kennen (wir wissen, wie man in einen Zug steigt, aber nicht, wo der Zug nach Mannheim abfährt), sei es, dass die an einem Ort erforderlichen oder erwünschten Handlungen gerade nicht für die dem Nutzer bekannten Routinen geeignet sind (an diesem Ort darf nicht geraucht werden), sei es, dass routinehaften Handlungen ausnahmsweise nicht möglich sind, also eine Sondersituation eintritt (der Aufzug bleibt stecken, die Straße ist blockiert). Schließlich ist es auch möglich, dass öffentliche Schrift überhaupt erst in bestimmte Handlungsmuster einführt, die eben keine Routinen sind (die Bedienung eines Straßenbahnfahrkartenautomaten in einer fremden Stadt etc.). Umgekehrt gilt: Wer sich in einem durch öffentliche Zeichen dicht strukturierten Raum bewegt, um lediglich die darin angebotenen Orte für routinisierte Handlungen zu nutzen und überdies diese Orte kennt, ignoriert die öffentlichen Zeichen: wer jeden Tag denselben Zug nimmt, braucht keine Schilder und Inschriften im Bahnhof, um sich zu orientieren, er nimmt sie gar nicht mehr zur Kenntnis.

Die ortsfeste Schrift eröffnet also Handlungs-Räume. Sie bietet dazu die Möglichkeit von Interaktion zwischen dem Zeichenproduzenten (der oft anonym ist) und dem Zeichenrezipienten und ersetzt damit Formen der face-to-face-Kommunikation, die unzuverlässig und störungsanfällig sind: wir könnten einen (Orts-)Kundigen fragen, wo sich das Rathaus befindet, ob man hier die Straße überqueren darf, wie man sein Gepäck aufbewahren kann oder wie die Straße heißt, in der wir uns befinden, aber nicht immer steht ein solcher Kundiger zur Verfügung, nicht immer spricht er unsere Sprache, nicht immer gibt er zuverlässig Auskunft.

Durch ihre Lösung von der Kopräsenz von Zeichenproduzenten und -rezipienten ähnelt die öffentliche Schrift bei aller Differenz der situationsgelösten geschriebenen Sprache, deren Funktionalität ebenfalls nicht an die Kopräsenz der Interaktionspartner gebunden ist. Dennoch würde das Bild 
der ,zerdehnten“ Sprechsituation (Ehlich 1984) in die Irre führen: ortsfeste Schrift ist oft depersonalisiert und immer von der Origo des Sprechzeitpunkts losgelöst. ${ }^{3}$ Während der Begriff der zerdehnten Kommunikation zwischen Autor und Leser auf die Zeitdimension fokussiert (in der gerade die mangelnde Kopräsenz zum Problem wird), ist die öffentliche Schrift von vorne herein zeitneutral angelegt, sie bezieht sich ganz auf den Ort.

\subsection{Indexikalische und nicht-indexikalische öffentliche Schrift}

Die Forschung über linguistische Landschaften untersucht meist (der oben zitierten Definition bzw. Aufzählung folgend) alle sichtbaren Schriftzeichen im öffentlichen Raum. Tatsächlich sind aber mehrere semiotische Typen zu unterscheiden, die es fraglich machen, ob diese Eigenschaft - öffentliche Sichtbarkeit - überhaupt einen kohärenten und sinnvollen Phänomenbereich eingrenzt. Vor allem sind längst nicht alle öffentlichen Schriftverwendungen in dem Sinn indexikalisch, dass sie nur an und durch den Ort interpretierbar sind, an dem sie sich befinden. Der Unterschied lässt sich schnell erkennen, wenn Zeichen „außer Betrieb“ gestellt werden. Das Schild in Abb. 1 mit der Aufschrift Achtung Rutschgefahr und einem entsprechenden Piktogramm ${ }^{4}$ kann nicht mehr interpretiert werden, weil es keinen Ort hat, auf den es sich bezieht. Seine Indexikalität ist vorübergehend außer Kraft gesetzt. Natürlich hat das Zeichen - wie alle indexikalischen Zeichen, z.B. die deiktischen Personalpronomen - immer noch ein Verweispotenzial: wir wissen, was es bedeuten würde, wenn es in Betrieb wäre. Die Indexikalität des Zeichens wird also nicht durch die Form des Zeichens selbst stillgelegt (wenn man einmal von Fällen semiotischer overlays im Zeichen selbst absieht, etwa dem ,Ausstreichen' eines Verkehrsschilds durch die kreuzweise darauf geklebte Folie); die Stilllegung muss vielmehr selbst kontextualisiert und kontextuell erschlossen werden. In Abb. 1 wird sie durch die Stapelung der identischen Schilder und ihre Aufbewahrung neben dem Reinigungsgerät, also auf der sozialen ,Hinterbühne' (Goffman 1959) eines Bahnhofs, erreicht. Beide sind außer Betrieb, die Schilder und das Reinigungsgerät. Das Schild wird offensichtlich von demselben Personal, das auch die Reinigungsmaschine bedient, im Bedarfsfall so aufgestellt, dass dadurch eine Warnung vor glatten Böden nach der Reinigung ausgesprochen wird.

\footnotetext{
3 Vermeintliche Gegenbeispiele wie die oft zitierten personalisierten und von einer (faktisch nicht gelieferten) Zeitorigo abhängigen Notizen des Typs bin gleich zurück sind gerade deshalb keine Gegenbeispiele, weil sie einen Fall stukturell missglückter Kommunikation darstellen und so zeigen, wie orts- und dingfeste Schrift gerade nicht funktionieren kann.

$4 \quad$ Das Schild selbst belegt übrigens gut die oben skizzierte These, dass öffentliche Schrift das Verhalten in bestimmten Räumen im Falle des Nicht-Greifens von Routinen steuert: wo man sonst routinemäßig geht, sollte man es nicht tun, wenn das Schild aufgestellt ist.
} 




Abb. 1: Ortsgebundene (indexikalische) Schilder außer Betrieb (stillgelegte Indexikalität) (Freiburg, Hauptbahnhof).

Neben solchen temporär stillgelegten ortsgebundenen Zeichen gibt es solche, die permanent außer Betrieb genommen worden sind, also nur noch geschichtliche Zeichen sind. Selbst dann aber ist ihre einstige Funktionalität meist noch lesbar, auch wenn ihre Referenz nicht mehr sicher ist. Die Inschrift auf dem Horn von Gallehus ist nicht mehr interpretierbar, weil wir ,Hlewegast' nicht kennen. Dennoch können wir davon ausgehen, dass er durch die Inschrift seinen Status als Auftraggeber gekennzeichnet hat. Die leere, im Jugendstil gestaltete Vitrine in der Haupteinkaufsstraße in Helsinki in Abb. 2 trägt die schwedische Inschrift $A / B$ Fazers-Musikhandel (ein Pendant auf Finnisch befindet sich auf demselben Gebäude etwas entfernt). Sie hat offensichtlich keine Referenz mehr, denn weder ist in der Vitrine etwas ausgestellt, noch gibt es an dieser Stelle der Alexanderinkatu ein Musikwarengeschäft. Die Schrift indiziert aber nach wie vor durch ihre Ding- und Ortsfestigkeit die Zugehörigkeit der Vitrine zu einem Geschäft, das es nicht mehr gibt. Über Inferenzen lässt sich erschließen, dass sich an diesem Ort die Musikhandlung Fazer befunden haben muss: das Schild ist historisch.

Innerhalb der Gruppe der indexikalischen öffentlichen Schriftzeichen sind weitere Untergliederungen möglich. So lassen sich indexikalische Verfahren identifizieren, die mit der Kontiguität zwischen Zeichen und intendiertem Referenten arbeiten. Dies trifft auf die eigentlich dingfesten Zeichen (Horn von Gallehus) zu, aber auch auf die Schilder in Abb. 1, die durch die 
Verbindung mit dem Boden, auf den sie stehen, Bedeutung erlangen. Daneben stehen Zeichen, die auf einen mehr oder weniger weit entfernten Ort verweisen; der Prototyp ist der distal zeigende Wegweiser (vgl. Abb. 3).

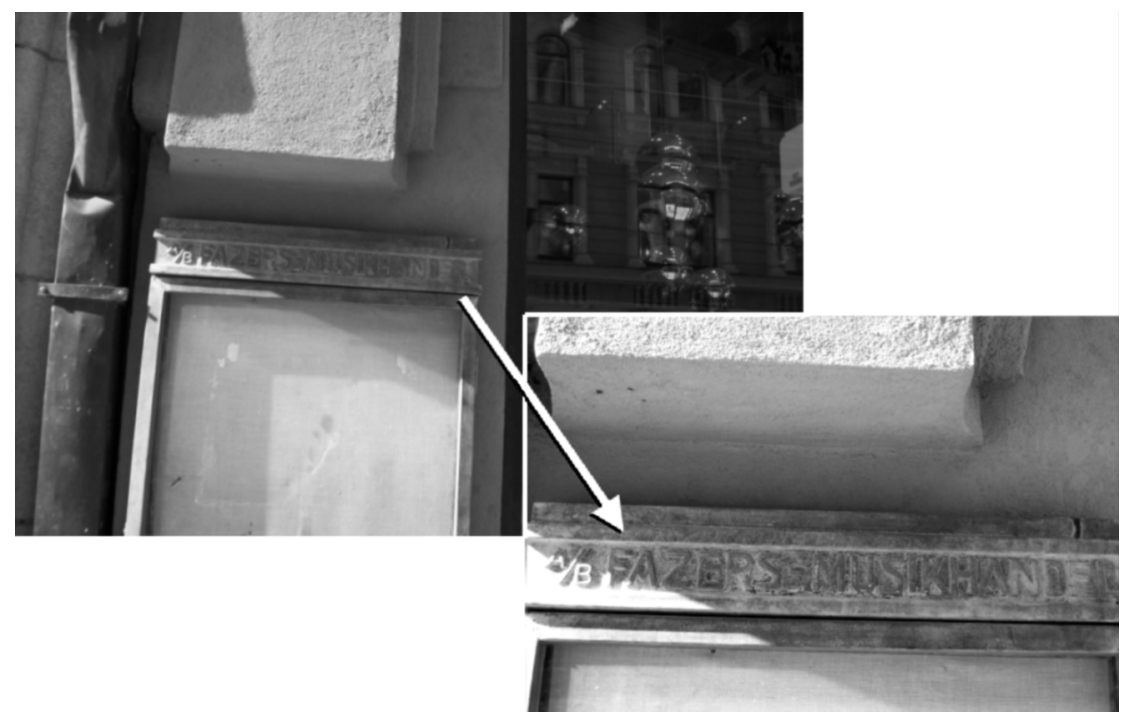

Abb. 2: Permanent stillgelegtes indexikalisches Zeichen: A/B Fazers Musikhandel (Helsinki, Alexanderinkatu; Foto K. Wagner).

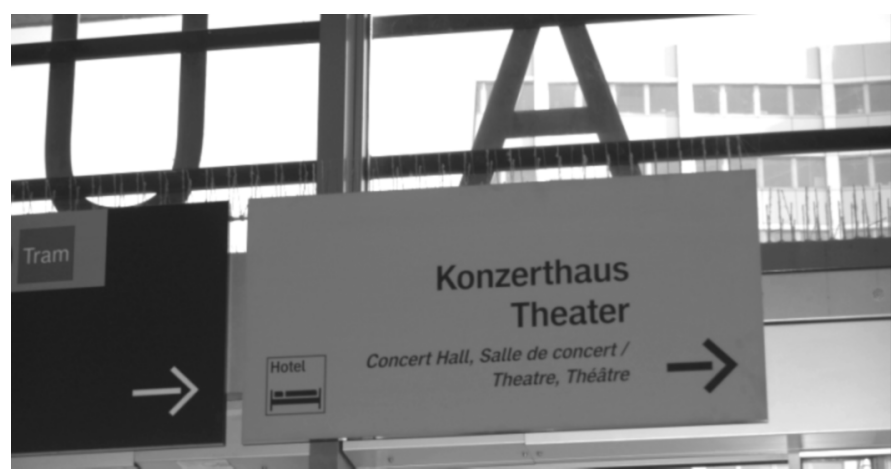

Abb. 3: Wegweiser (distaler Pointer), hohe Granularität, mehrsprachig deutschfranzösisch-englisch (Freiburg, Hauptbahnhof).

Das Beispiel aus Abb. 1 zeigt, dass ein indexikalisches Zeichen nicht auf ein spezifisches indexikalisches Sprachelement (wie „dieses Horn“) oder einen nichtverbalen pointer (den Pfeil in Abb. 3) angewiesen ist. Die Platzierung des Schilds mit der Aufschrift Achtung Rutschgefahr und dem entsprechenden icon (ausrutschendes Männchen) würde allein durch ihre räumliche Kontiguität mit dem Untergrund, auf den es sich bezieht, Bedeutung erlangen. 
(Dasselbe gilt für die Beispiele 2, 6, 7, 8, 9, 13-17.) Daraus ergibt sich als weitere Kategorisierungsmöglichkeit von indexikalischen Zeichen die Präsenz eines solchen pointers.

Schließlich lassen sich indexikalische Zeichen nach der Art subklassifizieren, in der sie sich auf den Ort beziehen. Die Identifizierung eines physikalischen Ort (wie in den bisher genannten Beispielen) als dem Thema der auf dem Zeichen verbalisierten rhematischen Information (,dieser Ort ist rutschig“, „dies ist eine Vitrine der Musikhandlung Fazer“ etc.) ist nur die einfachste Form dieses Bezugs. In anderen Fällen evoziert die öffentliche Schrift durch den Ort, an den sie angebracht ist, Wissensbestände, die für die Interpretation des Schriftzeichens unabdingbar sind. Etwa ist der Aufkleber auf dem in Abb. 12 dokumentierten Schild (Parkhauskasse: Automat steht im Parkdeck. kurz vor der Ausfahrt) deshalb indexikalisch, weil er eine ganze Handlungssequenz aufruft, die mit dem Benutzen des Parkhauses, an dessen Eingang er angebracht ist, verbunden ist. (Vgl. zu einem weiteren Beispiel Fußnote 5.)

Die Bedeutung öffentlicher, aber nicht indexikalischer, also nicht ortsgebundener und/oder dingfester Zeichen wird durch Veränderungen in der Platzierung hingegen nicht in derselben Weise berührt wie die indexikalischer. Sie können verstellt, abmontiert, in ihrer Richtung verändert werden, ohne dass sie ihre Funktion verlieren. Zu den nicht-indexikalischen Sprachzeichen im öffentlichen Raum gehört in der Regel die Werbung, aber auch transgressive (siehe unten, Abschnitt 2.8) Zeichen wie der kleine Aufkleber auf der Rückseite eines öffentlichen Verkehrszeichens in Abb. 4 mit der Aufschrift Schäuble is watching you - Stasi 2.0 und dem Bild des ehemaligen Innenministers des Bundes sowie viele Grafitti (Ausnahme sind die sog. Marken). Solche Zeichen können an vielen, oft an beliebigen Orten angebracht oder aufgestellt werden und sind überall in derselben Weise bedeutungsvoll und verständlich. ${ }^{5}$ Aus diesem Grund kann Werbung auch auf mobilen Objekten (Busse, Züge) angebracht werden. Das heißt natürlich nicht, dass solche nicht ortsgebundenen Zeichen nicht ebenfalls den Raum kolonialisieren und die Sprache territorialisieren, wie später noch ausgeführt wird (Abschnitt 2.8). Auch sind nicht-indexikalische öffentliche Zeichen an manchen Orten passender als an anderen; ein Reklameposter kann in einer Abstellkammer stehen, ein Werbeplakat im Museum hängen. Dann fehlt

\footnotetext{
Es gibt durchaus Fälle, in denen der Unterschied zwischen indexikalischer und nicht-indexikalischer öffentlicher Schrift nicht trivial ist, sondern eine genaue textuelle Analyse erfordert. Zum Beispiel sind die gedruckten Fahrpläne, die in deutschen Bahnhöfen aushängen, als Gesamttext kontextfrei verständlich. Die notwendige Information über die Gültigkeit des Fahrplans (,Sommerfahrplan 2009“) und den Ort, von dem die Züge abfahren oder wo sie ankommen, ist im Text selbst enthalten. Der Aushang ist funktional einer Version als Faltblatt oder „Städteverbindung“ äquivalent. Die ebenfalls funktional identischen Angaben auf den inzwischen üblichen elektronischen Anzeigetafeln über die abgehenden Züge sind hingegen indexikalisch in Zeit und Ort eingebunden.
} 
dem Zeichen sein intendierter Adressatenkreis. Dieser fehlende Kontext ist aber kein räumlicher. Die Zeichen sind in einer anderen Weise ,außer Betrieb" als die genannten indexikalischen Zeichen, denn sie konstituieren keinen Raum und sind von keinem Raum abhängig.

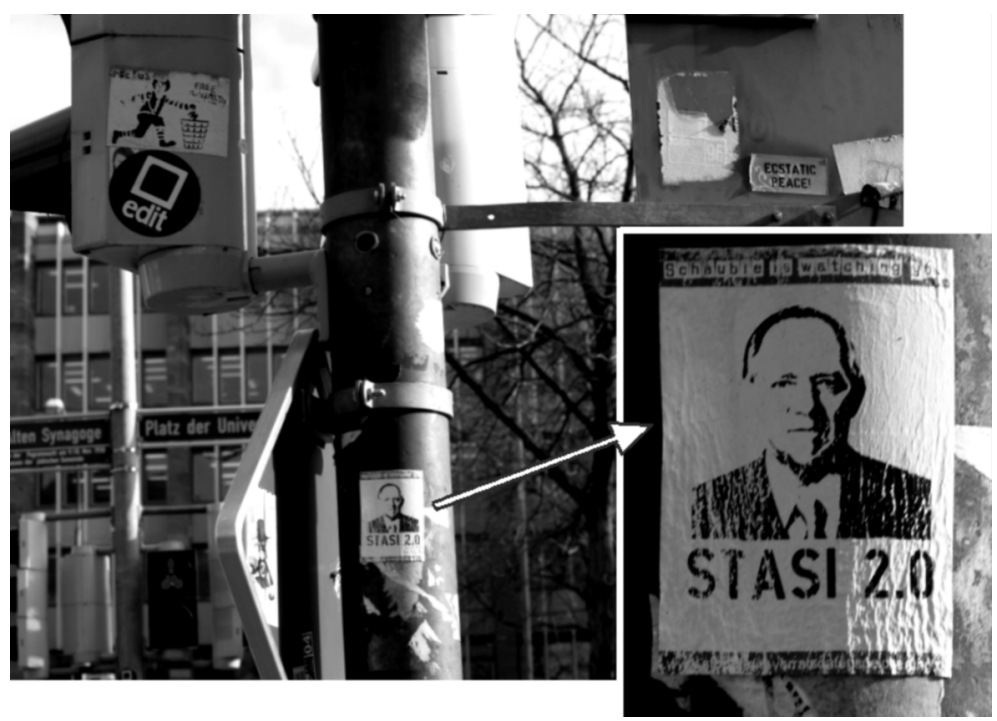

Abb. 4: Transgressives Zeichen, nicht-indexikalisch (Freiburg, Platz der Universität).

\subsection{Granularitätsstufen}

Ein zweites Manko der augenblicklichen Forschung über linguistic landscapes ist die Vernachlässigung der Granularität der öffentlichen Schriftzeichen. Nicht alle Schrift im öffentlichen Raum ist für dieselben Rezipienten gemacht; diese Rezipientenorientierung spiegelt sich in der Granularität des Zeichens wider. Sie bringt die intendierten Leser ins Spiel; allerdings nicht als Individuen, sondern in ihren sozialen Rollen. Diese Rollen sind in die Struktur des Zeichens eingeschrieben.

Hohe Granularität (also geringe Auflösung) ist zur allgemeinen und oberflächlichen Orientierung im Raum ausreichend. Ihr dienen Zeichen, die aus großer Entfernung gelesen werden können und die übergeordneten Handlungszielen (wie einen bestimmten Ort zu finden oder ihn mit einer bestimmten typischen Handlung zu identifizieren) zugeordnet sind (vgl. Abb. 3). Die intendierten Rezipienten dieser Zeichen sind in der Regel alle (nicht orts-kundigen) Menschen, die im öffentlichen Raum unterwegs sind. Geringe Granularität schränkt entweder die Gruppe der intendierten Rezipienten ein und selegiert dazu spezifischere soziale Rollen der Adressaten, oder sie dient subsidiären Handlungen innerhalb eines größeren Hand- 
lungssschemas. Solche Zeichen sind also nur für diejenigen Benutzer relevant, die sich bereits großflächig im Raum orientiert haben und nun spezifischere Informationen benötigen.

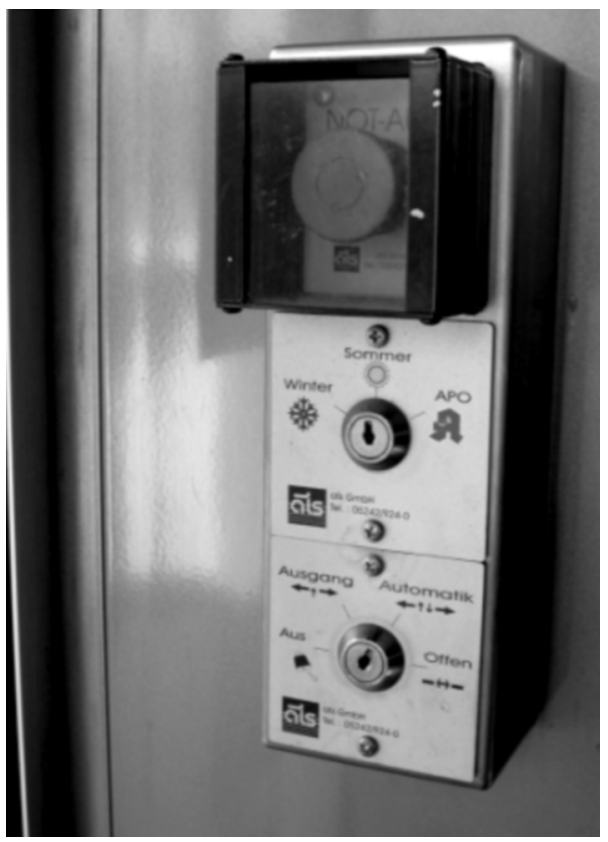

Abb. 5: Schließautomatik am Hauptportal des Freiburger Hauptbahnhofs: Zeichen mit geringer Granularität, das sich an spezifische Benutzergruppen richtet. Drei verschiedene Schilder in einem Ensemble und als Teil zweier Diskurse (Notfall und Bedienung).

Im ersten Fall haben wir es mit öffentlicher Schrift zu tun, die Handlungsräume eröffnet, die Spezialisten vorbehalten sind, z.B. dem Personal einer Institution oder Handwerkern, die sich in Notfällen im Raum orientieren müssen. Ein Beispiel ist in Abb. 5 zu sehen; die Anweisung zur Einstellung und Bedienung der automatischen Türen eines Bahnhofs per Hand ist sehr klein an der Bedienungsautomatik angebracht. Die normalen Benutzer sind weder interessiert noch berechtigt, diese Automatik zu bedienen. Besonders klein sind die Angaben über die herstellende Firma; sie selegieren nicht nur die spezialisierte Gruppe der Bahnhofsangestellten als soziale Adressaten, sondern sind überdies auch für sie nur in einem spezifischen Fall relevant, nämlich wenn ein Wartungstechniker der Firma zu informieren ist.

Im zweiten Fall werden öffentliche Schriftzeichen geringer Granularität im Zusammenhang einer einmal begonnenen Handlungsdurchführung gebraucht. Während zum Beispiel im Bahnhof Schilder mit hoher Granularität die Orientierung innerhalb des Gebäudes sowie den Weg aus dem Gebäude in die Stadt ermöglichen, sind Schilder geringer Granularität auf den Gepäcktrolleys angebracht (vgl. Abb. 6). Man kann sie erst aus der Nähe lesen, d.h. wenn man dabei ist, die subsidiäre Handlung des Gepäcktransports durch einen Gepäckwagen in Angriff zu nehmen. Noch kleiner 
- und in noch geringerer Granularität wieder auf einen Ausnahmefall hin orientiert - ist die Aufschrift auf diesem Schild, die Anweisungen gibt, wie man sich zu verhalten hat, wenn man das so beschilderte Objekt außerhalb des Bahnhofs findet. (Am kleinsten ist der TÜV-Stempel des DB-Geräteservice, der die Kontrolle der Funktionstüchtigkeit des Trolleys belegt.)



Abb. 6: Schild (und dreisprachiger Aufkleber englisch-deutsch-italienisch: please press bitte Bügel drücken pregasi premere) geringer Granularität für subsidiäre und Notfall-Handlungen (Freiburg, Hauptbahnhof). Die Abstufung der Schriftgrößen spiegelt die Spezialisierung der schriftlichen Hinweise wider (Nutzungsangabe im Normalfall/im Sonderfall/Aufkleber „TÜV“). Das Logo und die Aufschrift „Die Bahn“ zeigen Zugehörigkeit an.

\subsection{Schilder, ${ }^{6}$ Inschriften, Zettel und Aufkleber}

Eine dritte Unterscheidung betrifft die Materialität der öffentlichen Schriftzeichen und die damit zusammen hängende Frage der Begrenzung von Zeichen, also die Frage, was eigentlich zu einem Zeichen gehört. Inschriften sind dingfest: sie können nur schwer von der Oberfläche des Objekts entfernt werden, auf das sie sich beziehen. Zu diesem Typ gehören die eingangs diskutierten historischen Beispiele, aber auch edukativ-adhortative

\footnotetext{
Das Grimmsche Wörterbuch sagt zu Schild: „schild vorm bause, zum zeichen des dort betriebenen gewerbes: schild eines bäckers, eines kaufmanns, eines wirtshauses; ladenschild, herbergsschild, wirtshausschild $u$. ähnl. diese gewerbezeichen nannte man schilde, weil sie zunächst nur embleme entbielten und so sich den wappen verglichen." Im Vergleich zu dieser Definition ist die hier verwendete weiter und rein formal.
} 
Inschriften wie Die Wabrbeit wird ench frei machen ${ }^{7}$ auf dem Hauptgebäude der Freiburger Universität oder Häusernamen wie in Abb. 7. Weit häufiger ist im heutigen Stadtbild das Schild, auf dem die Schrift angebracht ist und das seinerseits mit dem Objekt direkt oder indirekt verbunden ist, auf das sich die Schrift bezieht. Das Schild ist ein Träger der Schrift, der zugleich durch seine Materialität die Grenzen des Zeichens markiert. Das Schild konstituiert also einen Rahmen, die bei der Inschrift gegebenenfalls erst geschaffen werden muss. Dadurch wird der so gerahmte Bereich als genau ein Zeichen konstituiert.

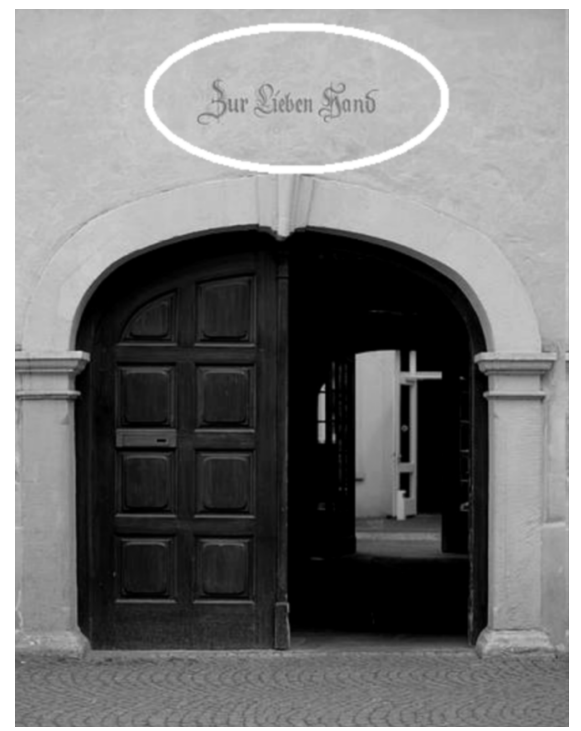

Abb. 7: Inschrift/Häusername (Freiburg, Innenstadt).

Dieses Zeichen kann allerdings komplex strukturiert sein (vgl. die Schilder in den Abb. 1, 3, 5, 6). Oft wird zum Beispiel Schrift mit nicht-sprachlichen Elementen kombiniert. Diese können ikonisch (Abb. 1) oder als pointer indexikalisch (im engeren Sinn des Wortes) sein. Pointer sind bei allen distal weisenden Zeichen (Wegweisern) obligatorisch (vgl. Abb. 3, 12). Mehrere durch ihre Materialität als Schilder konstituierte Einzelzeichen können aufeinander Bezug nehmen. So ist in Abb. 8 ein Ensemble (siehe unten Abschnitt 2.5) aus drei Schildern (Zeichen) abgebildet, wie sie für die heutige Verkehrsbeschilderung typisch ist. Das oberste, rein piktogrammatische

Der indexikalische Charakter dieser Inschrift mag nicht unmittelbar evident sein; wie eng die Bedeutung dieses Mottos mit dem Typ von Gebäude zusammenhängt, auf dem es angebracht ist, wird aber deutlich, wenn man die völlig andere Bedeutung im ursprünglichen $\mathrm{Zu}$ sammenhang (nämlich Joh 8,32) zum Vergleich heranzieht (dazu Kaiser 2003). Während im Johannes-Evangelium die Wahrheit mit Christus identifiziert wird, ist sie auf der Universität gerade aufklärerisch anti-klerikal gemeint. Diese Uminterpretation macht den so bestimmten sozialen Raum in einer ganz anderen Weise sozial nutzbar als das Bibelwort es täte. Sie konstituiert den akademischen Raum gegen die Kirche. 
Schild weist die Straße als Fussgängerzone aus; das darunter angebrachte Schild verwendet Schriftsprache, um die Gültigkeit des obersten Schilds auf die Zeiten des zweiwöchentlichen Marktes einzuschränken; schließlich nimmt ein drittes schriftsprachliches Schild Fahrradfahrer von dieser Einschränkung aus.

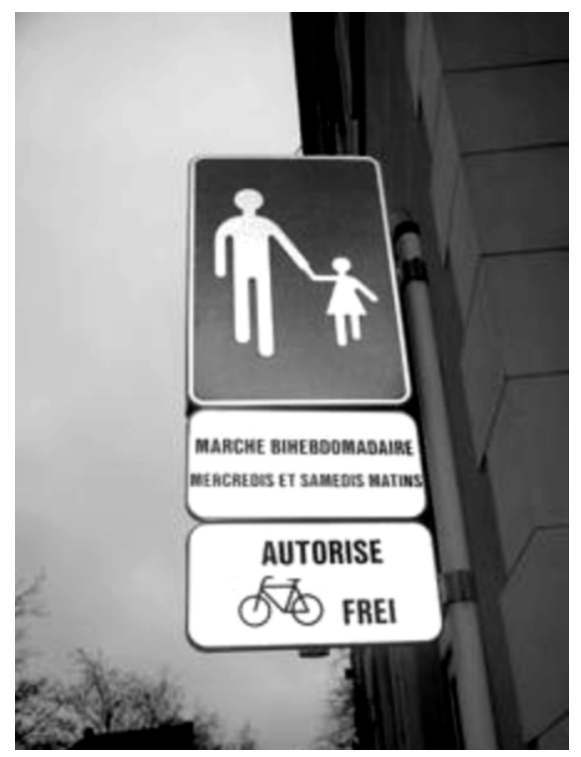

Abb. 8: Ensemble von drei Schildern aus einem Diskurs, eines davon mehrsprachig(deutsch/französisch). Funktion: Verbot bestimmter Raumnutzungen (regulatives Zeichen) (Luxemburg Stadt, Place Guillaume II).

Anders als bei Schildern ist die Grenze (der Rahmen) einer Inschrift oft problematisch; die Beantwortung der Frage, ob es sich um ein einzelnes, komplex strukturiertes, oder um mehrere Zeichen handelt, muss auf vielfältige gestalterische und inhaltliche Aspekte Bezug nehmen. In Abb. 9 ist es aus beiden Gründen naheliegend, die benennende Inschrift (Palladium) sowie die Inschrift zur Funktionsangabe (café \& tanzpalais) als ein Zeichen zu verstehen. In Abb. 10 ist es hingegen offen, ob die englische Inschrift auf der linken Eingangstür eines Geschäfts für Mobiltelefone in Helsinki als ein unabhängiges Zeichen zu verstehen ist, dem die finnische Inschrift auf der rechten Eingangstür als komplementäres, aber getrenntes Zeichen innerhalb eines Ensembles zugeordnet ist, oder ob beide Inschriften als Teil desselben Zeichens zu verstehen sind.

Zwischen Schildern und Aufschriften steht die Hybridkategorie des Aufklebers. Er ist in seiner Materialität gut abgegrenzt und präsentiert sich daher als ein Zeichen. Andererseits ist er in der Regel nur schwer von dem Objekt zu lösen, auf dem er angebracht ist. Dies machen sich gerade transgressive Aufkleber (vgl. Abb. 4) zunutze. Zettel, die angeheftet sind, sind temporäre Schilder, deren Flüchtigkeit gerade durch ihre gering Haftung betont wird. 


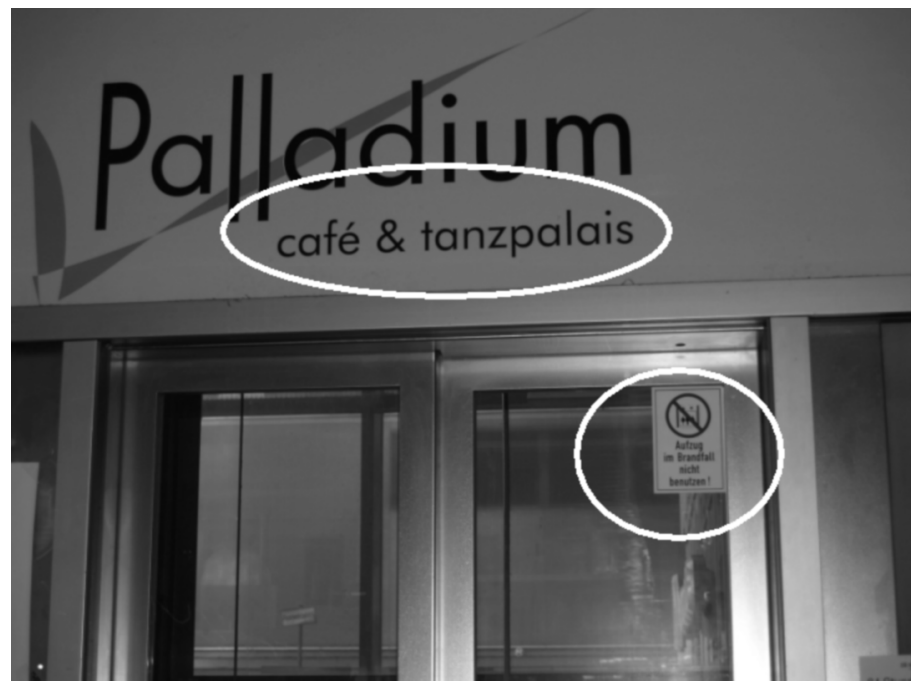

Abb. 9: Inschrift aus zwei Komponenten und Aufkleber (Freiburg, Hauptbahnhof). Funktion: Gebrauchsweise eines Orts (Palladium), Gebrauchsverbot eines anderen (Aufzug).

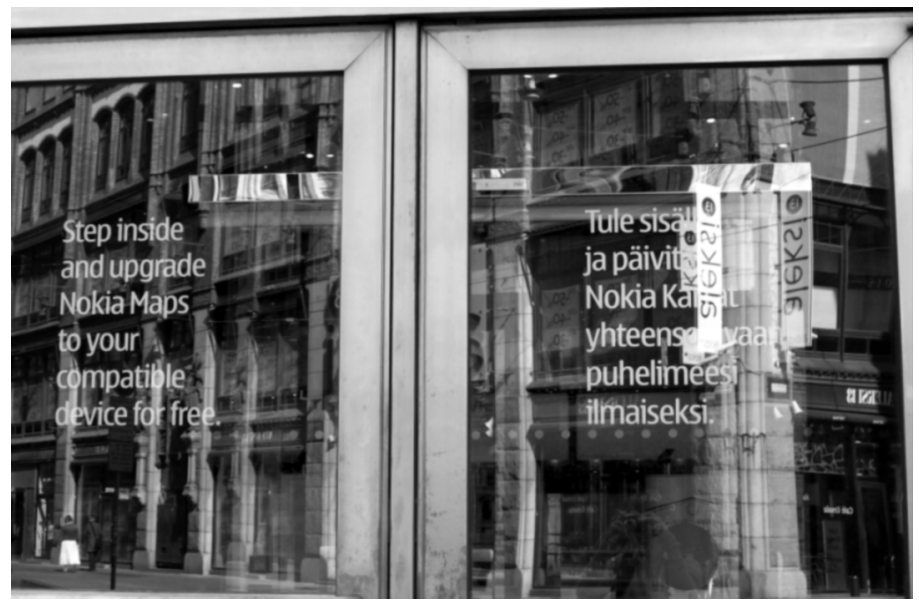

Abb. 10: Zwei Inschriften = zwei Zeichen? (Helsinki, Alexanderinkatu, Foto K. Wagner). Die linke Inschrift (Step inside and upgrade Nokia Maps to your compatible device for free) ist eine Übersetzung der rechten (Tule sisälle ja pä̈vitä Nokia kartat ybteensopivaan pubelimeesi ilmaiseksi).

\subsection{Ensembles, Diskurse, (Über-)Schichtungen}

Wie bereits gezeigt, stehen öffentliche, Schrift enthaltende Schilder, Aufkleber oder Inschriften oft nicht allein, sondern beziehen sich aufeinander. Dabei sind drei Arten der Bezugnahme zu unterscheiden. 
Unter einem Ensemble verstehe ich mehrere Schilder/Inschriften/ Zettel/Aufkleber, die in unmittelbarer Nähe zueinander stehen, also mit einem Blick wahrgenommen werden können, und die sich inhaltlich aufeinander beziehen. Ein typisches, schon diskutiertes Beispiel findet sich in Abb. 8. Davon ist eine zweite Art der Bezugnahme im öffentlichen Raum zu unterscheiden, in der nicht die Kopräsenz der Schilder/Inschriften/Aufkleber, sondern ihre formale und funktionale Ähnlichkeit ausschlaggebend ist. Ich spreche hier von einem Zeichendiskurs. ${ }^{8}$ Die Zeichen, die zu einem Diskurs gehören, sind typischerweise nicht zusammen sichtbar; der Zeichenrezipient nimmt aber die einzelnen Zeichen eines Diskurses in ihrer gegenseitigen Kohärenz und Bezugnahme war. Die Grundlage dieser diskursiven Kohärenz ist oft die formale Beschaffenheit des Zeichens (sein Design), die seinerseits auf einen identischen Zeichenproduzenten hinweist. Es gibt aber auch andere Diskurse konstituierende Faktoren wie Kontiguität (Ketten von Wegweisern, die den Weg zu einem Ziel definieren).



Abb. 11: Zeichen-Ensembles, Zeichen-Diskurse. Die hoch-granulären Wegweiser gehören einem anderen Diskurs an (= Radfahren in Freiburg) als die niedriggranulären (Europa-Fahrradwanderwege $3=$ Mulhouse/ Freiburg und 2 = Colmar/Freiburg; Fahrradwanderwege $\mathrm{Mg}=$ Markgräfler Land, $\mathrm{Ds}=$ Dreisamtal, $\mathrm{Br}=$ Breisgau $)$.

Abb. 11 zeigt beide Formen von Bezugnahmen. Alle Schilder bilden ein Ensemble, bei dem die Interpretation der kleineren Schilder rechts von der Pfeilrichtung des größeren Wegweisers darüber abhängen. Sie gehören aber drei verschiedenen Diskursen an. Einerseits gibt es zwei Wegweiser mit Ziel-

8 Der Begriff wird auch von Scollon/Scollon (2003) gebraucht, allerdings in einer weiteren Bedeutung. 
angaben innerhalb von Freiburg, die sich durch das identische Design und die identische Funktion (Orientierung für Radfahrer in der Stadt) als Mitglieder desselben Diskurses ausweisen, andererseits (etwas kleiner) zwei blaue Schilder mit abgerundeten Ecken, die Langstrecken-(Europa-)Fahrradwege nach Frankreich markieren, schließlich rechts davon drei kleinere farbige Schilder, die regionale Langstrecken-Fahrradwege in die Region beschildern. Diesen drei Diskursen schließen sich nicht nur die genannten, sondern zahlreiche andere Schilder an anderen Orten an, die dasselbe Design und dieselbe semiotische Struktur aufweisen.

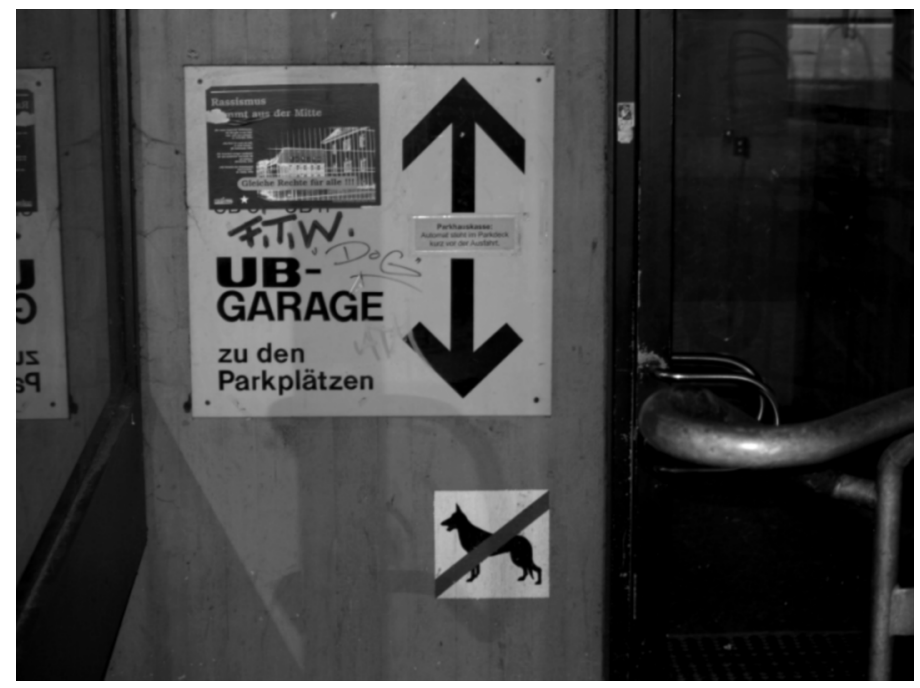

Abb. 12: Überschichtung von Zeichen (Freiburg, ehemalige UB-Garage). Die überschichtenden Zeichen sind teils indexikalisch (Aufkleber: Parkhauskasse: Automat stebt im Parkdeck kurz vor der Ausfabrt), teils nichtindexikalisch (= der transgressive Aufkleber Rassismus kommt aus der Mitte ... und Marken).

Eine dritte Art von Bezugnahme zwischen öffentlichen Schriftzeichen lässt sich am besten als (Über-) Schichtung (layering, Scollon/Scollon 2003) bezeichnen. Überschichtungen entstehen dann, wenn sekundäre Aufkleber und Aufschriften auf primären Zeichen angebracht werden. Erneut ist zwischen indexikalischen und nicht-indexikalischen überschichtenden Zeichen zu unterscheiden. Überschichtungen bringen den für öffentliche Schrift ungewöhnlichen Aspekt der Zeit ins Spiel: sie implizieren eine Trennung zwischen einem früheren (primären) und späteren (sekundären, tertiären ...) Zeichen. Beispiele reichen von autorisierten Ergänzungen und Hinweisen (etwa dem Aufkleber heute! auf einem Veranstaltungsplakat) bis zu transgressiven Zeichen, die öffentliche Zeichen in nicht autorisierter Weise karikieren oder subvertieren. In Abb. 12 ist das primäre Zeichen ein Schild, das 
vor dem Eingang in das Parkhaus der Universitätsbibliothek angebracht wurde. Es zeigt in Verbindung mit zwei Pfeilen den Weg ins Parkhaus, das man über Treppen nach oben und unten nach Betreten des Gebäudes erreichen kann. Zwischen den beiden Pfeilen ist - offensichtlich improvisiert ein dennoch autorisierter Aufkleber angebracht, der einen weiteren Hinweis gibt: Parkhauskasse: Automat stebt im Parkdeck kurz vor der Ausfahrt. Es handelt sich dabei um eine Überschichtung; das ursprüngliche Schild wurde später durch eine spezifischere Angabe zu einer subordinierten Handlung (Zahlen vor der Ausfahrt aus dem Parkhaus) ergänzt, ganz offensichtlich, weil die Benutzer des Parkhauses an diesem Eingang vergeblich nach einem Kassenautomaten suchten. Sowohl das primäre als auch das sekundäre Zeichen sind indexikalisch (wenn auch, wie bereits ausgeführt, in verschiedener Weise und in unterschiedlichen Granularitätsstufen). Darüber hinaus ist auf dem primären Zeichen jedoch ein weiterer Aufkleber zu sehen, der von einer Antifa-Gruppe stammt, die sich für gleiche Rechte für ausländische Häftlinge einsetzt. Dieses overlay ist kein indexikalisches Zeichen; es bezieht sich weder auf das primäre Zeichen noch ist es in seiner Interpretation vom Standort (oder Träger) abhängig. Dasselbe gilt für die ebenfalls in Überschichtung angebrachten Marken (F.T.W., dog, etc.).

\subsection{Die Grammatik ding- und ortsfester Schriftsprache}

Angesichts der fast ausschließlichen Beschäftigung mit Texten in Buchform und den entsprechenden schriftsprachlichen Grammatikstrukturen hat die Linguistik es bisher weitgehend versäumt, die typischen Strukturen dingund ortsfester Schriftsprache herauszuarbeiten und als Ergebnis der spezifischen Funktionalität dieser Schriftverwendung zu interpretieren. Es ist aber ganz offensichtlich, dass ding- und ortsfeste Schrift genauso wie mündliche Sprache und ortsungebundene Schriftsprache strukturelle Eigenschaften aufweist, die ihrer spezifischen Funktionalität geschuldet sind (vgl. dazu auch Schmitz in diesem Band). Schrift im öffentlichen Raum - besonders wenn sie hohe Granularität aufweist - hat normalerweise nicht viel Platz; sie soll aus der Distanz sichtbar sein. Hieraus ergeben sich Formen sprachlicher Indexikalität, die ihrerseits durch lokales (im wörtlichen Sinn: am Ort des Zeichens aufzufindendes) oder Weltwissen aufgefüllt werden müssen. Wir finden im öffentlichen Raum grammatische Konstruktionen, die in der situationsungebundenen Schriftsprache (wie auch in der gesprochenen Sprache) selten sind und daher oft als „randgrammatisch“ bezeichnet werden. Faktisch handelt es sich aber um häufige und problemlos interpretierbare Muster, die durchaus zum Zentrum unseres grammatischen Wissens gehören. Ein gutes Beispiel sind die modalen (sog. deontischen) Infinitive wie

\footnotetext{
$9 \quad$ Den Begriff verwendet Fries (1987) in einem sehr lesenswerten Artikel zu solchen Kon-
} struktionsformaten. 
Aufzug im Brandfall nicht benutzen! (Abb. 9)

Bitte Bügel drücken! (Abb. 6)

die ihre pragmatische Kraft ohne alle Morphologie eindeutig (wenn auch kontextabhängig) entwickeln können (vgl. die ausführliche Analyse in Deppermann 2007, Kap. 2), aber auch zahlreiche andere verblose oder nominalisierte Konstruktionen, wie

Bei Betreten der Gleise Lebensgefahr

Schwimmweste unter dem Sitz.

Verteiler Notstromaggregat

Achtung Rutschgefahr! (Abb. 1)

Zu den Parkplätzen (Abb. 12)

Für Ibr Gepäck - nur zur Benutzung im Bahnhof (Abb. 6)

Frei (Abb. 8)

Klärungsbedürftig ist insbesondere die Frage, wie diese im Vergleich zur nicht ortsgebundenen Schriftsprache grammatisch recht reduziert erscheinenden Konstruktionen im Verwendungskontext ohne Reste von Ambiguität erfolgreich funktionieren. Die Antwort ergibt sich aus einer Mischung zwischen Form/Funktion-Kopplung und Empraxis (Bühler 1934 [1982]). Form/Funktion-Kopplung bedeutet, dass bestimmte grammatische Konstruktionen mit bestimmten Zeichenfunktionen gekoppelt sind. Zum Beispiel beziehen sich deontische Infinitive in der Regel auf Objekte und Orte, die bestimmte Handlungen nahe legen. Diese Handlungen werden in der einen oder anderen Weise näher bestimmt oder eingeschränkt. Es findet also eine Kopplung zwischen der Funktion „Gebrauchsweisen vorschlagen/verbieten" (siehe 2.7, unten) und der Konstruktion statt, die gerade deshalb auf Angaben zu Zeit, Person, Modus verzichten kann (sie sind im Infinitiv neutralisiert). Andere Form/Funktion-Koppelungen sind die Konstruktion Achtung NP, die sich mit Zeichen verbindet, die Warnungen aussprechen, sowie die Konstruktion „z $2 u+$ def.Art $+\mathrm{N}$ “, die sich mit Wegweisern verbindet.

Die zweite Ressource, die die Zeichennutzer verwenden, um grammatisch einfache Formen eindeutig zu verstehen, ist die von Bühler so genannte empraktische Nennung (1934 [1982], S. 157-159), also die Tatsache, dass in bestimmten Situationen der Handlungskontext bereits so starke Vorgaben macht, dass eine sprachliche Explizierung verschiedener Situationsparameter nicht mehr nötig ist. Das betrifft in unserem Fall sicherlich den Adressaten, der das Zeichen rezipiert (ihm durch Blick zugewandt ist) und daher nicht mehr genannt werden muss. Darüber hinaus sind aber in der Situa- 
tion, in der das Zeichen rezipiert wird, oft auch schon bestimmte Handlungen naheliegend bzw. das Spektrum an Handlungsalternativen ist stark eingeschränkt. So ist die Beschilderung

\section{Für Ibr Gepäck}

empraktisch eingebunden in einen Handlungsablauf, den der Leser schon (zu planen) begonnen hat, wenn er vor dem Gepäcktrolley steht; dieser ist damit als Gegenstand der Charakterisierung eingeführt (der Adressat wird aus Höflichkeitsgründen, nicht aus Gründen der referenziellen Desambiguierung, dennoch in possessiver Form expliziert). In

\section{nur zur Benutzung im Babnhof}

fehlt in der Nominalisierung der Adressat, ebenso wie wiederum die Explizierung des Objekts der Benutzung. Die genaue Interpretation solcher öffentlichen Schriftverwendung ist allerdings trotz aller empraktischer Einbindung oft von kulturellen Wissensbeständen abhängig. So ist die ₹u-Konstruktion nicht nur oft auf Wegweisern anzutreffen, sie kommt auch mit der Funktion ,Benennung' auf Gasthausschildern und auf Häusern vor (Hausnamen). Um zu verstehen, dass das Schild in Abb. 7 nicht als Wegweiser dient, um den Besucher zur lieben Hand zu führen, sondern das Haus benennt, über dessen Eingang es steht, andererseits aber die Präpositionalphrase zu den Parkplätzen auf dem Schild in Abb. 12 nicht etwa den Namen des Parkhauses darstellt, sondern den Weg durch die Tür zu den Parketagen weist, erfordert Erfahrungswissen über typische Hausnamen, über die Unwahrscheinlichkeit, dass ein Parkhaus einen Namen trägt, über die Kopplung von Schrift und Pfeil etc. Selbst dieses Erfahrungswissen reicht jedoch nicht immer aus. Der Aufkleber Beide Scheiben einschlagen über den Notausgangsfenstern der ICE-Züge und neben einem roten Hammer ist nur verständlich, wenn man über das technische Wissen verfügt, dass ICE-Fenster doppelverglast sind und sich unter der zunächst eingeschlagenen Scheibe eine weitere befindet.

\subsection{Funktionen ortsfester Zeichen}

Die Funktionen öffentlicher Schrift sind grundsätzlich beschränkt. Es scheint, dass sich die im öffentlichen Raum verwendeten indexikalischen Zeichen, die Schriftsprache enthalten, exhaustiv folgenden Funktionsbereichen zuordnen lassen: 1) Benennen und Charakterisieren, 2) Zugehörigkeit markieren, 3) Gebrauchsweisen vorschlagen oder verbieten, 4) Wege weisen sowie 5) Ermahnen und Gedenken.

1) Benennen ist eine grundlegende Funktion öffentlicher Schrift, die in der heutigen Zeit vor allem der Orientierung dient. Benannt werden Bauwerke, vor allem aber Straßen und Plätze. Das typische Beispiel sind Häusernamen, 
die mit den Aufkommen von Straßennamen und Hausnummern zwar nicht mehr nötig wären, bei bedeutenden und großen Gebäuden (nicht zuletzt als Landmarken innerhalb der Stadt) aber weiterhin üblich sind. Benannt werden aber auch Abbildungen von Personen (etwa auf einem Denkmal oder einem Grabstein) sowie manchmal die Personen selbst; das berühmteste Beispiel ist die Beschilderung des gekreuzigten Iesus mit INRI, Namensschilder bei Kongressen oder auf Uniformen gehören in dieselbe Kategorie.

Das Benennen durch Eigennamen wie in den bisher genannten Fällen lässt sich auf das Benennen durch Gattungsnamen (Kategorisierung) erweitern. Hierzu gehören Produktnamen (Boeing 737 auf einem Flugzeug, Vaio auf einem Laptop), aber selbst ganze Objektkonstellationen; etwa wird der Inhalt eines Schaufensters auf Abb. 13 durch den finnisch-schwedisch bilingualen Aufkleber suvi - sommar auf dem Schaufenster zu einer Darstellung des Sommers (Sommermode, Picknick-Korb, Erdbeeren) zusammengefasst. Von solchen einfachen Kategorisierungen sind andere Verwendungen von Gattungsnamen zu unterscheiden, die nicht der Benennung dienen, sondern schon Angaben über mögliche oder unmögliche Handlungen implizieren (vgl. die Funktion 3 unten).

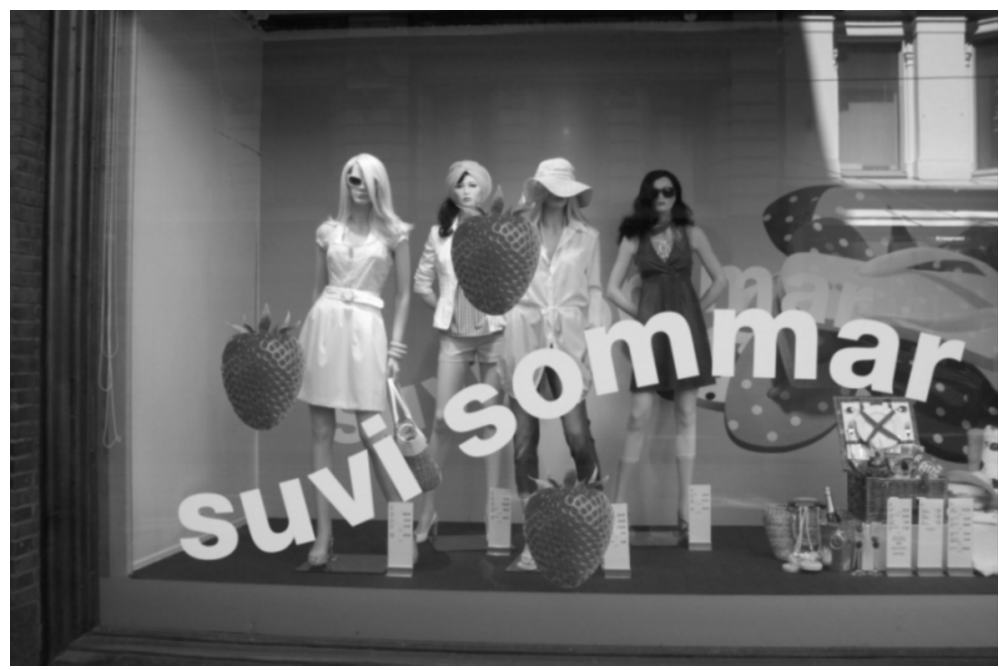

Abb. 13: Benennung eines Schaufensterinhalts durch einen Gattungsnamen (finnisch swi $=$ schwedisch sommar ,Sommer) (Helsinki, Stockmans, Foto K. Wagner).

Benannte Dinge, Personen, Orte und Institutionen werden gern durch Epitheta weiter charakterisiert: Iesus durch Herkunft und (ironischen) Titel (Iesus Nazarenus Rex Iudaeorum), Geschäfte durch eine Angabe zu ihrem Alter (seit 1911), Menschen durch Titel und Status (Dr. Müller, Abteilungsleiter, auf einem Namensschild), etc. 


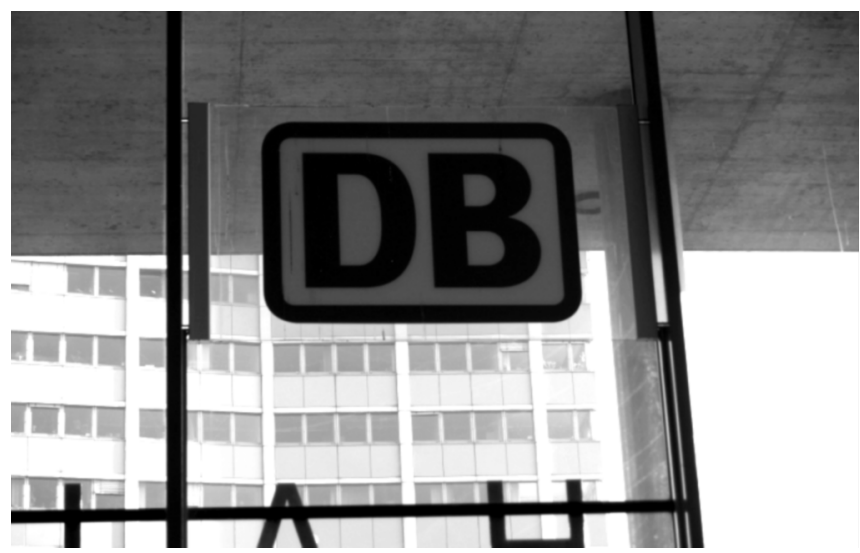

Abb. 14: Das große über dem Haupteingang angebrachte Logo der Deutschen Bahn markiert das Territorium des Konzerns und die Zugehörigkeit des Gebäudes zur Bahn (Freiburg, Hauptbahnhof).

2) Schrift im öffentlichen Raum vermittelt zudem Relationen zwischen Orten und (natürlichen oder juristischen) Personen, sie markiert also Zugehörigkeit. Die Art der Zugehörigkeit kann vielfältig sein. In einer wichtigen Form der Zugehörigkeitsmarkierung geht es um Besitz; die Schriftzeichen fungieren dann als Marken wie auf Abb. 14. Da der Besitz oft in seiner räumlichen Ausdehnung markiert wird, wird durch solche Schriftverwendung ein Territorium gekennzeichnet. In Abb. 14 gehört das so markierte Gebäude zum Beispiel zum Territorium der Deutschen Bahn (DB). Andere Zugehörigkeitsrelationen sind die des Erbauers, Architekten oder Finanziers; auf vielen Gegenständen in der Öffentlichkeit findet sich auch der Name und die Adresse des produzierenden Handwerkers. Es gibt auch sehr spezifische Zugehörigkeitsrelationen, die durch öffentliche Schrift hergestellt werden; etwa die Versicherung eines Gebäudes gegen Brand durch eine Plakette (die die Zugehörigkeit zu dieser Schutzgemeinschaft indiziert). Die Trennung zwischen reiner Zugehörigkeitsangabe und Indizierung einer Gebrauchsweise für das Objekt sind fließend. So wird auf dem Steuerungsinstrument in Abb. 5 einerseits durch die Angabe des Herstellers (ATS GmbH) eine Zugehörigkeitsrelation hergestellt (produzierende Firma), andererseits wird durch die Angabe der Telefonnummer auch eine Gebrauchsweise im Fall des Defekts der Türöffnungsanlage vorgeschlagen.

3) Während Benennung und Zugehörigkeitsmarkierung indirekt Handlungspotenziale eröffnen können (das durch den Namen identifizierte Wirtshaus ist das, in dem man einkehrt; der genannte Architekt bekommt einen neuen Auftrag ...), ist es heute die wichtigste Funktion öffentlicher, ortsfester Schrift, direkte Angaben zum Gebrauch eines Orts zu machen oder bestimmte Handlungen zu verbieten oder einzuschränken. Funktions- 
bezeichnungen finden sich auf vielen öffentlichen Gebäuden (Mädchenbildungsanstalt, Service point, Bäckerei). Dabei kommen jedoch nicht nur einfache Bezeichnungen vor, sondern auch mehr oder weniger ausführliche Angaben darüber, was an dem jeweiligen Ort zu finden ist (z.B. bei Restaurants in Form von Speisekarten), welche Öffnungszeiten gelten, wie Geräte zu bedienen sind etc. Zum Beispiel zeigt Abb. 9 einerseits den Eingang zu einem Lokal namens Palladium (Benennung), andererseits wird durch die kleiner (und damit untergeordnet) hinzugefügten Wörter Café \& Tanzpalais indiziert, in welcher Weise der so benannte Raum genutzt werden kann. Zu den Verboten gehören viele Verkehrsschilder (wie in Abb. 8), Verbote des Zutritts oder der Benützung von Aufzügen im Brandfall (wie in Abb. 9), etc. Manche Vorschläge für die Verwendung von Räumen sind sehr ausführlich; man vergleiche die Aufforderung auf dem Mobilfunkgeschäft in Helsinki (Abb. 10) Step inside and upgrade Nokia maps to your compatible device for free / Tule sisälle ja päivitä Nokia kartat ybteensopivaan pubelimeesi ilmaiseksi; andere sind knapp; vgl. den einfachen Aufkleber Ale Pabeier - Altpapier - Vieux papier auf einer Mülltonne in Abb. 15.

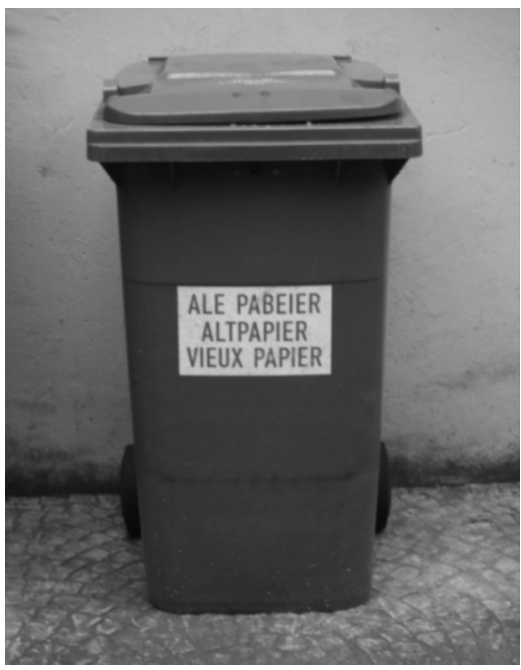

Abb. 15: Funktion Gebrauchsanweisung: Mülleimer mit dreisprachigem Aufkleber (Luxemburgisch, Deutsch, Französisch) in Luxemburg Stadt.

4) Die Funktion, Wege zu weisen, gehört sicherlich zu den wichtigsten und ältesten der ortsfesten Schrift. Dabei kann sowohl das Ziel benannt werden (vgl. Abb. 11, die weißen Schilder, Abb. 3) als auch der Weg (vgl. die farbigen Schilder auf Abb. 11). Wegweiser verweisen oft auf Orte, die nicht im visuellen Umfeld des Zeichenrezipienten sind, sie sind also nicht auf die visuelle Identifizierbarkeit des indizierten Objekts oder Orts angewiesen (der Pfeil fungiert als distaler pointer). Dabei darf man den Begriff Wegweiser nicht zu eng verstehen; auch in Abb. 12 wird dem Nutzer des Parkhauses der Weg gewiesen. 




Abb. 16: Öffentliche Schrift (Inschrift) in der Funktion des Gedenkens (Luxemburg Stadt, Denkmal für den Autor des „Renert“, einer Adaption von Goethes „Reineke Fuchs“ in luxemburgischer Sprache).

5) Öffentliche Schrift kann schließlich dazu dienen, vergangener Ereignisse oder verstorbener Menschen zu gedenken (Abb. 16), und sie kann zu moralisch richtigem Verhalten ermahnen (Abb. 17 und 18).
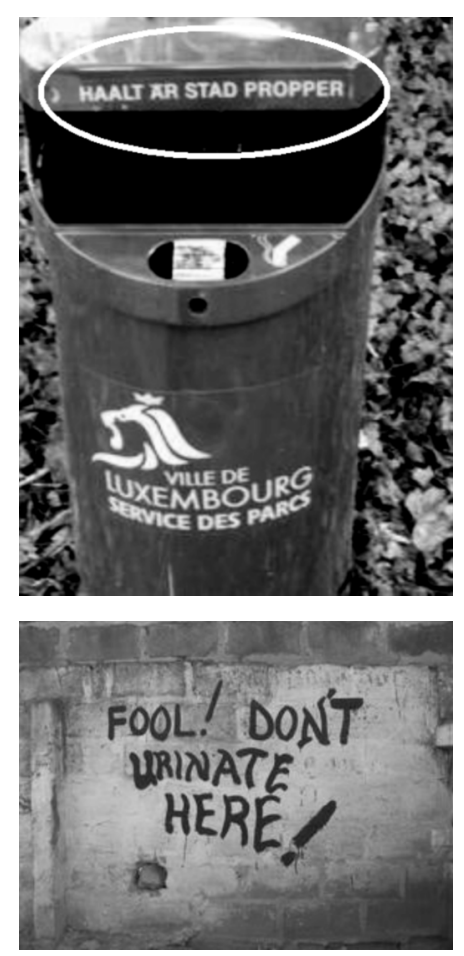

Abb. 17: Öffentliche Schrift (Inschrift) in der Funktion der Ermahnung (haalt är Stad propper = , haltet Eure Stadt sauber') (Luxemburg Stadt).

Abb. 18: Öffentliche Schrift (Inschrift) in der Funktion der Ermahnung: transgressives oder autorisiertes Zeichen? (Ghana, Accra). 


\subsection{Die Kolonialisierung des öffentlichen Raums durch die Schrift}

Die bisherige Forschung zu linguistischen Landschaften vernachlässigt nicht nur, wie sich in die schriftsprachlichen Zeichen die Art des potentiellen Rezipienten einschreibt, sie ignoriert oft auch die Seite des Zeichenproduzenten und damit die Formen von Agentivität und Macht, die mit der Gewalt über die Zeichenverwendung im öffentlichen Raum einher gehen. Es ist offensichtlich, dass die Möglichkeiten, im öffentlichen Raum durch Sprachzeichen Orte für soziale Handlungen zu konstituieren oder dort bestimmte Handlungen zu verbieten, nicht jedem Gesellschaftsmitglied gleichermaßen offensteht. In der Forschung wird oft - einem Vorschlag von Ben-Rafael et al. (2006, S. 14f.) folgend - zwischen ,top-down'-Zeichen (,issued by national and public bureaucracies - public institutions, signs on public sites, public announcement and street names") und „bottom-up“Zeichen, die von Privatpersonen geschrieben und angebracht werden, unterschieden. Diese Unterscheidung verkennt aber, dass die Ordnungsprinzipien, die die Gestaltung des öffentlichen Raums regulieren, die öffentlichen und privatwirtschaftlichen Agenten nicht auf ein Oben vs. Unten abbilden. Vielmehr wird die semiotische Gestaltungsmacht über Raumsegmente von zwei Prinzipien reguliert: nach dem einen Prinzip erwerben die (privaten) Besitzer von Raumsegmenten für diese Teile des Raums Gestaltungsprivilegien, nach dem anderen Prinzip behalten die öffentlichen (staatlichen) Institutionen Gestaltungsmacht über den gesamten übrigen, nicht in Privatbesitz befindlichen Raum.

Die im öffentlichen Raum sichtbaren Zeichen symbolisieren die Macht der Agenten, die sie angebracht oder aufgestellt haben; die Segmente des Raums, die die staatlichen und privatwirtschaftlichen Agenten dabei für sich beanspruchen, teilen den öffentlichen Raum annähernd vollständig auf, d.h. sie kolonialisieren ihn. Zeichen, die weder dem Prinzip des privaten Raumbesitzes noch dem Prinzip des öffentlich-staatlichen Raumprivilegs folgen, sind heutzutage automatisch transgressiv: sie sind ganz offensichtlich ohne Autorisierung angebracht und haben keine offizielle Autorität. Transgressive Zeichen konstituieren deshalb einen Gegendiskurs schon allein dadurch, dass sie die Kolonialisierung des öffentlichen Raums durch bestimmte Agenten staatlicher oder privater Art durchbrechen. Ihre reine Existenz ist angesichts der vollständigen Aufteilung des Raums unter seinen privatwirtschafltichen und staatlichen Kolonialisatoren provokativ. Oft sind sie klein und unauffällig und werden von der mainstream-Gesellschaft gar nicht wahrgenommen. Vorzugsweise werden sie an abseitigen Stellen angebracht, auf Rück- und Hinterseiten, auf stillgelegten und heruntergekommenen Gebäuden (vgl. Abb. 4, 12). Dass sie oft nur dem geübten Blick sichtbar werden und nur dem eingeweihten Blick verständlich sind, rückt 
sie in die Nähe alter subkultureller Zeichensysteme wie der Gaunerzinken, von denen sie sich allerdings dadurch unterscheiden, dass sie in der Regel nicht indexikalisch sind. ${ }^{10}$ Der transgressive Wirkungsgrad transgressiver Zeichen steigt, je mehr sie von der allgemeinen Öffentlichkeit nicht mehr ignoriert werden können.

Die Autorität eines Zeichens ergibt sich nur selten direkt aus der gesellschaftlichen Position seines Autors, denn öffentliche Zeichen sind gerade dadurch gekennzeichnet, dass kein spezifischer, persönlicher Autor zu identifizieren ist, der für sie verantwortlich ist. (Nur wenige Schilder sind wie die klischeehaften Verbotsschilder auf deutschen Spielplätzen mit autorisierenden Autorenangaben wie „Der Hausbesitzer“ versehen.) Es ist die Struktur des Zeichens selbst, die seine Autorität zu einem wesentlichen $\mathrm{Ma} ß$ garantieren muss. Um autoritative Zeichen zu verfassen, müssen Regeln eingehalten werden. Grosso modo gilt: je größer der technische Aufwand, mit dem das Zeichen hergestellt wurde, je größer also die Kosten, die seine Herstellung verursacht hat, je länger damit aber auch die Zeitdauer, für die es gelten soll, um so größer ist die Autorität des Zeichens. Improvisierte Zeichen (z.B. Zettel) haben deshalb weniger Autorität als industriell gefertigte, handgeschriebene noch weniger. Tatsächlich ist hier die Grenze zur Transgressivität fließend. Bei der in Abb. 18 gezeigten handgesprühten Inschrift auf einer Mauer in Accra/Ghana, weiß man nicht, ob es sich um ein transgressives Zeichen handelt, oder ob es legitim vom Besitzer dieses Raums angebracht wurde, der nur nicht über die Ressourcen verfügte, um dem Zeichen eine autoritativere Form zu geben. Vielleicht handelt es sich sogar um einen gesellschaftlichen Kontext, in dem der öffentliche Raum noch nicht so vollständig kolonialisiert ist, dass individuelle Agenten nur transgressiv in ihm tätig werden können. Der Autor wird sicherlich einen Grund gehabt haben, diese Inschrift anzubringen; ihr improvisierter Charakter lässt ihr aber (zumindest in unseren Augen) keine große Autorität zukommen.

\section{Abschließende Bemerkungen}

Ziel dieses Beitrags war es, einige grundlegende Überlegungen zur Analyse ortsfester Schrift anzustellen, die in unserem öffentlichen Leben eine große Rolle spielt. Dabei wurden einige Parameter entwickelt, die neben der semiotischen Struktur des Zeichens selbst, seiner Materialität und seiner Granularität, auch den Produzenten und Rezipienten des Zeichens ins Blickfeld rücken. Der öffentliche Schriftgebrauch erhält damit eine pragmatische Dimension.

10 Hierzu gehört auch der große Bereich der Graffiti, die allerdings zum großen Teil nicht sprachlich sind und deshalb hier nicht ausführlich berücksichtigt werden (vgl. etwa von Treeck 2003; Meier 2007). Graffiti sind indexikalisch, wenn sie als tags Raum territorialisieren und als Marken fungieren. 
Der primäre Gegenstand der heutigen Forschung über linguistic landscapes, nämlich die öffentliche Repräsentation der Sprachen einer mehrsprachigen Gesellschaft, ist damit noch gar nicht ins Blickfeld geraten, und in der Tat scheint es sinnvoll, solche Fragen als sekundäre Anwendungsfelder eine Forschungsrichtung zu sehen, die zunächst wesentlich grundlegendere Fragen zu klären hätte. Dennoch lässt sich nicht übersehen, dass alle Zeichen im öffentlichen Raum, die Sprache enthalten, einen wesentlichen Effekt haben: sie territorialisieren Sprache(n) nämlich, d.h. sie weisen Sprache(n) geografische Räume zu, in denen sie Gültigkeit beanspruchen können. Diese Gültigkeit haben bei nicht-transgressiven Zeichen im offiziellen Raum fast ausschließlich den Staats- und/oder Amtssprachen der betroffenen Länder. In offiziell mehrsprachigen Ländern wie Luxemburg (Abb. 8, 15) oder Finnland (Abb. 10, 13) sind die öffentlichen Schilder und Inschriften demselben Prinzip entsprechend mehrsprachig und symbolisieren damit die Gültigkeit von mehr als einer Sprache auf einem Territorium. $\mathrm{Zu}$ den Amts- bzw. Staatssprachen tritt in vielen Ländern die internationale lingua franca Englisch (Abb. 3, 4, 6), nur selten weitere große Verkehrssprachen wie Französisch (Abb. 3) oder gar Italienisch (Abb. 6). Nicht offiziell anerkannte Sprachen, zum Beispiel die von Immigrantengruppen, kommen abgesehen von transgressiven Zeichen fast nur in Verbotsschildern (vgl. Hinnenkamp 1989) sowie auf den privaten, in der Regel unprofessioniellen Schildern und Zetteln des Kleinhandels vor. Sie können in der Regel kein eigenes Territorium auf dem Gebiet der Mehrheitssprache(n) beanspruchen. Die Schrift im öffentlichen Raum trägt also wesentlich zur Territorialisierung von Sprachen bei: sie macht die Zuordnung von Räumen und Sprachen sichtbar. Der vorliegende Beitrag sollte allerdings auch zeigen, dass diese Zuordnung sich nur im größeren Zusammenhang einer Theorie der Schrift im öffentlichen Raum verstehen lässt, die es noch zu entwickelt gilt.

\section{Literatur}

Auer, Peter (2009): Visible dialect. In: Hovmark, Henrik/Stampe Sletten, Iben/Gudiksen, Asgerd (Hg.): I mund og bog. 25 artikler om sprog tilegnet Inge Lise Pedersen på 70-årsdagen d. 5. juni 2009. Kopenhagen, S. 31-46.

Ben-Rafael, Eliezer/Shohamy, Elana/Hasan Amara, Muhammad/Trumper-Hecht, Nira (2006): Linguist landscape as symbolic construction of the public space: The case of Israel. In: International Journal of Multilingualism 3, 1, S. 7-30.

Bühler, Karl (1934 [1982]): Sprachtheorie. Jena. [Ungek. Neudr. d. Ausg. Jena 1934. Stuttgart 1982].

Coulmas, Florian (2008): Linguistic landscaping and the seed of the public sphere. In: Shohamy/Gorter (Hg.), S. 13-24. 
Deppermann, Arnulf (2007): Grammatik und Semantik aus gesprächsanalytischer Sicht. (= Linguistik - Impulse und Tendenzen 14). Berlin.

Ehlich, Konrad (1984): Zum Textbegriff. In: Rothkegel, Annely/Sandig, Barbara (Hg.): Text - Textsorten - Semantik. Hamburg, S. 9-25.

Fries, Norbert (1987): Zu einer Randgrammatik des Deutschen. Zur Theorie randgrammatischer satzwertiger Konstruktionen. In: Meibauer, Jörg (Hg.): Satzmodus zwischen Grammatik und Pragmatik. (= Linguistische Arbeiten 180). Tübingen, S. 75-95.

Giddens, Anthony ([1979] 1995): Strukturierung und sozialer Wandel. In: Müller, HansPeter/Schmid,Michael (Hg.): Sozialer Wandel. Modellbildung und theoretische Ansätze. Frankfurt a.M., S. 151-191. [Originalausg. 1979].

Giddens, Anthony (1984): The constitution of society. Cambridge.

Goffman, Erving (1959): The presentation of self in everyday life. New York.

Hausendorf, Heiko (i.Dr.): Zwischen Linguistik und Literaturwissenschaft: Textualität revisited. In: Zeitschrift für germanistische Linguistik (ZGL) 36.

Hinnenkamp, Volker (1989): Interaktionale Soziolinguistik und Interkulturelle Kommunikation. Tübingen.

Kaiser, Gerhard (2003): Die Wahrheit wird euch frei machen: die Freiburger Universitätsdevise - ein Glaubenswort als Provokation der Wissenschaft. In: Wenzler, Ludwig (Hg.): Welche Wahrheit braucht der Mensch? Freiburg i.Br., S. 47-103.

Koß, Gerhard (2002): Namenforschung: eine Einführung in die Onomastik. 3., aktual. Aufl. (= Germanistische Arbeitshefte 34). Tübingen.

Landry, Rodrigue/Bourhis, Richard (1997): Linguistic landscape and ethnolinguistic vitality: an empirical study. In: Journal of Language and Social Psychology 16, 1, S. 23-49.

Meier, Stefan (2007): Stylelife - Graffiti als typografisches Ausdrucksmittel sozialen Stils. In: Kimminich, Eva/Rappe, Michael/Geuen, Heinz/Pfänder, Stefan (Hg.): Express yourself! Europas kulturelle Kreativität zwischen Markt und Underground. (= Cultural Studies 25). Bielefeld, S. 193-208.

Ong, Walter J. (1982): Orality and literacy. The technologizing of the word. London.

Schmauks, Dagmar (2002): Orientierung im Raum. Zeichen für die Fortbewegung. (= Probleme der Semiotik 20). Tübingen.

Scollon, Ron/Scollon, Suzie Wong (2003): Discourse in public places - language in the material world. London.

Shohamy, Elana/Gorter, Durk (Hg.) (2008): Linguistic landscape: expanding the scenery. London/New York.

Treeck, Berhard von (2003): Typografie als Mittel zur Identitätsbildung. In: Androutsopoulos, Jannis (Hg.): HipHop: Globale Kultur - Lokale Praktiken. Bielefeld, S. 102-120. 